\title{
SYK correlators for all energies
}

\author{
Alexandre Streicher \\ Institute for Advanced Study, Princeton, NJ 08540, U.S.A. \\ Perimeter Institute for Theoretical Physics, Waterloo, ON N2L 2Y5, Canada \\ Stanford Institute for Theoretical Physics, Stanford University, Stanford, CA 94305, U.S.A. \\ Department of Physics, University of California, Santa Barbara, CA 93106, U.S.A. \\ E-mail: streicher@ias.edu
}

Abstract: The Sachdev-Ye-Kitaev (SYK) model, a theory of $N$ Majorana fermions with $q$-body interactions (a.k.a. $q$-local), becomes in the large $q$ limit a conformally-broken Liouville field theory. Taking this limit preserves many interesting properties of the model, yet makes the theory as a whole much more tractable. Accordingly, we produce novel expressions for the two and four-point correlators at arbitrary temperature and find the surprising result they take a universal closed form. We note that these expressions correctly match onto and interpolate between previously-obtained low-energy results and simple high-energy perturbative checks. We find that the time-ordered four-point correlators are always determined by finite temperature OPEs into the identity and Hamiltonian, while the out-of-time-order four-point correlators remain nontrivial and always scramble. This has only been established in the conformal limit, so to find that it holds for large $q$ at all temperatures/couplings is a nontrivial result. Finally, we determine the system's thermalization and scrambling rates and find that they always agree, regardless of temperature. This adds to the increasing body of evidence that there exists simple underlying structures in the dynamics of $q$-local systems, such as those formed by SYK's epidemic operator growth.

Keywords: AdS-CFT Correspondence, Conformal Field Theory, Holography and condensed matter physics (AdS/CMT), 1/N Expansion

ArXiv ePrint: 1911.10171 


\section{Contents}

1 Introduction 1

2 Large $q$ SYK $\quad 3$

2.1 Large $q$ approximation 3

2.2 Twisted two-point function 4

2.3 Two-point correlators at all energies 4

3 Four-point correlators at all energies $\quad 5$

3.1 Time-ordered correlators and finite temperature OPEs 5

$\begin{array}{ll}3.2 & \text { Out-of-time-order correlators and chaos }\end{array}$

4 Discussion $\quad 8$

$\begin{array}{lr}\text { A Twisting correlators } & 9\end{array}$

B Fundamental domain of four-point functions $\quad 12$

\section{Introduction}

The Sachdev-Ye-Kitaev model is one of the only solvable models of many-body chaos, containing $N$ flavors of Majorana fermions all coupled together in $q$-body interactions (a.k.a. $q$-local) $[1,2]$. The model is made tractable by taking an large $N$ t'Hooft limit, which results in the theory becoming dominated by the infinite "melonic" subset of planar diagrams, rather than the intractable set of all planar diagrams often found in matrix models [3]. In such a large $N$ limit, the low-energy physics was found to saturate a chaos bound on the decorrelation often out-of-time-order (OTO) four-point correlators [4-6], hinting at a rich underlying structure for this model that at first glance does not contain any notion of spatial locality.

This physics was shown to arise from the near-conformal low-energy behavior of the model, where fluctuations are universally governed by a Schwarzian action [5, 7, 8]. Such a one-dimensional action naturally arises by recasting $2 D$ Jackiw-Teitelboim gravity into its $1 D$ boundary holographic action [7]. Alternatively, one may take a $1 \ll q \ll N$ limit of the SYK model to arrive at an effective description in terms of broken $1 D$ Liouville field theory, whose action is only a field redefinition away from that of the Schwarzian [9]. Indeed, after taking the t'Hooft limit, this large $q$ limit makes the model even more tractable, as the physics of broken Liouville theory is much easier to analyze than generic broken conformal physics. 
Recently this limit was used to find details of $S Y K$ operator evolution, where a microscopic wavefunction amongst the many-body degrees of freedom spread in a manner matching that of a primary operator "falling" along higher weight operators/descendants (or dually, how such a near-boundary operator propagates deeper into the bulk), when evolved using one of the non-compact time-like generators of the $1 D$ conformal group $[10,11]$. Furthermore, they were able to determine that this behavior was universal in the large $q$ limit, regardless of temperature/energy, so the graph-theoretic epidemic intuition from infinite temperature evolution remained relevant [12].

In appendix A we review how two-point functions "twisted" to satisfy Euclidean-time bi-local boundary conditions generate higher correlators. Of course, it is extremely difficult to solve for such an object in a generic theory, as even determining the regular two-point function may be impossible. However, by utilizing the solvability of the broken Liouville large $q$ limit of the SYK model, we are able to find such a "twisted" two-point function. Turning the crank, we obtain the large $q$ two-point correlator as well as the time-ordered and out-of-time-ordered four-point correlators for arbitrary energies and couplings. We confirm that these expressions correctly match onto both weak-coupling/high-temperature perturbative results and previously-derived strong-coupling/low-temperature expressions [5]. We have thus produced the first set of resummed expressions for four-point correlators at arbitrary energies.

There exist several interesting phenomena as one varies the energy and/or coupling. First we note that at all energies, the time-ordered four-point function is entirely determined by finite temperature OPEs into the identity and Hamiltonian, which has only previously been shown to hold in the near-conformal limit [5]. That is, for all intents and purposes, when there are no other operators between two flavor-averaged fermions on the thermal circle, one has that to first nontrivial order in $N$

$$
\hat{G}_{12} \equiv \frac{1}{N} \sum_{i=1}^{N} \hat{\psi}_{i}\left(\tau_{1}\right) \hat{\psi}_{i}\left(\tau_{2}\right)=\left\langle\hat{G}_{12}\right\rangle_{\beta} \hat{\mathbb{I}}+\frac{\left\langle\delta \hat{H} \hat{G}_{12}\right\rangle_{\beta}}{\left\langle(\delta \hat{H})^{2}\right\rangle_{\beta}} \delta \hat{H}
$$

where $\delta \hat{H} \equiv \hat{H}-\langle\hat{H}\rangle_{\beta}$ is the Gram-Schmidt-processed Hamiltonian. Such a simple expansion does not hold for the OTO four-point correlators, which signals that the large $q$ limit does not just reduce the theory to a free theory, but rather preserves interesting interactions. In fact, we find that all of the OTO four-point correlators scramble, regardless of the energy, with the same rate as that of thermalization

$$
\lambda_{L} \sim \frac{2}{t_{\text {thermal }}} \sim \frac{2 \pi}{\beta} v \quad \pi v=\beta \mathcal{J} \cos \frac{\pi v}{2}
$$

where $v=v(\beta \mathcal{J})$ is determined by the energy and coupling of the system [5]. Of course, the two timescales are parametrically separated by $\log N$, but it is still interesting to note that thermalization and scrambling occur at the same rate for all energies and couplings and not just in the near-conformal limit. The matching of these two rates has only been observed in systems such as large $c 2 D C F T$ s where the temperature controls all rates [13]. 


\section{Large $q$ SYK}

The SYK model features $q$-local interactions with independently random couplings, where each of the couplings is normal distributed

$$
H=i^{q / 2} \sum_{1 \leq i_{1} \ldots \leq i_{q} \leq N} J_{i_{1} \ldots i_{q}} \psi_{i_{1}} \ldots \psi_{i_{q}} \quad\left\langle J_{i_{1} \ldots i_{q}}^{2}\right\rangle=\frac{J^{2}}{\left(\begin{array}{c}
N-1 \\
q-1
\end{array}\right)}=\frac{\mathcal{J}^{2}}{2 q\left(\begin{array}{c}
N-1 \\
q-1
\end{array}\right)} \quad\left\{\psi_{i}, \psi_{j}\right\}=2 \delta_{i j}
$$

At large $N$, the two-point function satisfies the saddle-point equations

$$
[G]^{-1}=\left[G_{0}\right]^{-1}-[\Sigma] \quad \Sigma\left(\tau_{1}, \tau_{2}\right)=\frac{\mathcal{J}^{2}}{2 q}\left(G\left(\tau_{1}, \tau_{2}\right)\right)^{q-1}
$$

where bracketed terms are Matsubara frequency matrices. One should note that since our fermions square to one, $\left[G_{0}\right]^{-1}=-i \omega / 2$ rather than typical $-i \omega$.

\subsection{Large $q$ approximation}

When generating diagrams using the Schwinger-Dyson equations, note that diagrams where melons are inserted into melons receive a combinatorial $q$ enhancement, as there are many rungs upon which one may insert (hence the need for a $q^{-1}$ factor in the self-energy to keep everything finite). However, diagrams where melons are simply threaded together do not receive this enhancement [14]. Thus, at large $q$ only the former dominate, which can be captured by the following truncation of the Schwinger-Dyson expansion:

$$
[G]=\left[G_{0}\right]+\left[G_{0}\right][\Sigma]\left[G_{0}\right] \quad \Sigma=\frac{\mathcal{J}^{2}}{2 q} G^{q-1}
$$

Combining the equations together and Fourier transforming, one obtains

$$
\partial_{\theta_{1}} \partial_{\theta_{2}}\left(G-G_{0}\right)=-\frac{2}{q}\left(\frac{\beta \mathcal{J}}{2 \pi}\right)^{2} G^{q-1}
$$

The role played by $G_{0}$ in this equation is to require that $G \rightarrow G_{0}$ as $\theta_{12}^{-}$goes to integer multiples of $2 \pi$. If we accordingly re-express $G=G_{0} e^{\sigma / q}$, then we obtain Liouville's equation

$$
\partial_{\tau_{1}} \partial_{\tau_{2}} \sigma=-2\left(\frac{\beta \mathcal{J}}{2 \pi}\right)^{2} e^{\sigma}+\mathcal{O}(1 / q)
$$

where the field $\sigma$ is expected to be periodic in both of its arguments, as well as have kinks when $\theta_{12}$ approaches integer multiples of $2 \pi$.

Rather than calculate the original SYK Green's function $G$, we would like to figure out how to solve Liouville's equation for two-point function $\mathcal{G}$ obeying the the constraint

$$
\lim _{\theta_{1} / \theta_{2} \rightarrow \theta_{3}^{+}}\left(\begin{array}{c}
\mathcal{G}\left(\theta_{1}, \theta_{2}\right) \\
\mathcal{G}\left(2 \pi-\theta_{1}, \theta_{2}\right)
\end{array}\right)=\left(\begin{array}{cc}
\cosh (\mu) & -\sinh (\mu) \\
-\sinh (\mu) & \cosh (\mu)
\end{array}\right) \lim _{\theta_{1} / \theta_{2} \rightarrow \theta_{3}^{-}}\left(\begin{array}{c}
\mathcal{G}\left(\theta_{1}, \theta_{2}\right) \\
\mathcal{G}\left(2 \pi-\theta_{1}, \theta_{2}\right)
\end{array}\right)
$$

In the large $q$ limit, the twist conditions decouple and become

$$
\lim _{\theta_{1} / \theta_{2} \rightarrow \theta_{3}^{+}} \sigma=\lim _{\theta_{1} / \theta_{2} \rightarrow \theta_{3}^{-}} \sigma-q \nu+\mathcal{O}\left(q^{-1}\right)
$$

where $\nu=\mu G\left(\theta_{34}^{-}\right)$. 


\subsection{Twisted two-point function}

Due to the presence of the operator insertions at $\theta_{3}$ and $-\theta_{3}$ (a.k.a. $2 \pi-\theta_{3}$ ) and the point reflection symmetry $\left(\theta_{12}^{-}, \theta_{12}^{+}\right) \rightarrow\left(2 \pi-\theta_{12}^{-}, 2 \pi-\theta_{12}^{+}\right)$(B.5), we need only solve for $\mathcal{G}$ in three regions of the domain $0<\theta_{12}^{ \pm}<2 \pi$. First, we have the uncrossed twisted correlators

$$
\mathcal{G}= \begin{cases}G_{0}\left(\frac{\sin \gamma_{1}}{\sin \left(v_{1} \theta_{12}+\gamma_{1}\right)}\right)^{2 / q} & \theta_{1}>\theta_{2}>\theta_{3} \\ G_{0}\left(\frac{\sin \gamma_{2}}{\sin \left(v_{2} \theta_{12}+\gamma_{2}\right)}\right)^{2 / q} & \theta_{3}>\theta_{1}>\theta_{2}\end{cases}
$$

and the crossed twisted correlator

$$
\begin{array}{r}
\mathcal{G}=G_{0} e^{-\nu}\left(\frac{\sin \gamma_{1} \sin \gamma_{2}}{e^{-q \nu} \sin \left(v_{1} \theta_{13}^{-}\right) \sin \left(v_{2} \theta_{23}^{-}\right)-\sin \left(v_{1} \theta_{13}^{-}+\gamma_{1}\right) \sin \left(v_{2} \theta_{23}^{-}-\gamma_{2}\right)}\right)^{2 / q} \\
\theta_{1}>\theta_{3}>\theta_{2}
\end{array}
$$

Furthermore, Liouville's equation (2.5) constrains the parameters

$$
\pi v_{i}=\beta \mathcal{J} \sin \gamma_{i}
$$

while the point reflection symmetry $\left(\theta_{12}^{-}, \theta_{12}^{+}\right) \rightarrow\left(2 \pi-\theta_{12}^{-}, 2 \pi-\theta_{12}^{+}\right)$(B.5) leads to more constraints

$$
e^{-q \nu} \sin \left(\frac{v_{1}}{2}\left(\pi-\theta_{3}\right) \pm \frac{v_{2}}{2} \theta_{3}\right)=\sin \left(\frac{v_{1}}{2}\left(\pi-\theta_{3}\right) \pm \frac{v_{2}}{2} \theta_{3}+\gamma_{1} \pm \gamma_{2}\right)
$$

Given particular values of $\theta_{3}, \beta \mathcal{J}$, and $\mu$ these four constraints (2.10) (2.11) uniquely determine $v_{1 / 2}$ and $\gamma_{1 / 2}$. Furthermore, when $\nu=\mu=0$, we have that $v_{1}=v_{2}=v$, $\gamma_{1}=\gamma_{2}=\frac{\pi}{2}(1-v)$, and that

$$
\pi v=\beta \mathcal{J} \cos \frac{\pi v}{2}
$$

\subsection{Two-point correlators at all energies}

By taking the $\mu \rightarrow 0$ limit of our twisted two-point function, we may recover the previouslyderived large $q$ expression for the two-point function at arbitrary temperature [5] in the domain $0<\theta_{12}^{ \pm}<2 \pi$ (with all other values determined by symmetries)

$$
G=G_{0}\left(\frac{\cos (\pi v / 2)}{\cos \left(v\left(\frac{\pi}{2}-\theta_{12}^{-}\right)\right)}\right)^{2 / q}=G_{0}\left(\frac{\pi v}{\beta \mathcal{J} \cos \left(v\left(\frac{\pi}{2}-\theta_{12}^{-}\right)\right)}\right)^{2 / q}
$$

We see then that the thermal timescale - the timescale of variation for the two-point correlator - is not given by what is expected for either a perturbative theory $\left(t_{\text {thermal }} \sim\right.$ $\mathcal{J}^{-1}$ ) nor that of a conformal theory or a theory lacking quasiparticles $\left(t_{\text {thermal }} \sim \beta\right.$ ). Instead, we find that the thermalization timescale interpolates between these two behaviors as it is given by

$$
t_{\text {thermal }} \sim \frac{\beta}{v} \sim \frac{1}{\mathcal{J}}\left(1+\frac{(\beta \mathcal{J})^{2}}{8}+\mathcal{O}\left((\beta \mathcal{J})^{4}\right)\right) \sim \beta\left(1+\frac{2}{\beta \mathcal{J}}+\mathcal{O}\left((\beta \mathcal{J})^{-2}\right)\right)
$$


in the perturbative/high-energy and strong-coupling/low-energy regime respectively. Furthermore, from the equation for $v(2.12)$, we see that the thermalization scale is strictly bounded above by $\beta$. We conclude no matter how large the coupling is, a conformal system will always thermalize faster.

\section{Four-point correlators at all energies}

We now have everything we need to obtain the four-point function. Defining the disconnected and connected four-point correlators $F \equiv F_{d}+\frac{\mathcal{F}}{N}$, we find that the connected four-point correlator is generated by the twisted two-point correlator

$$
2 \lim _{\nu \rightarrow 0} \partial_{\nu} \ln \mathcal{G}\left(\theta_{1}, \theta_{2}\right)=\frac{\mathcal{F}\left(\theta_{1}, \theta_{2}, \theta_{3},-\theta_{3}\right)}{G\left(\theta_{12}^{-}\right) G\left(\theta_{34}^{-}\right)}=\frac{\mathcal{F}\left(\theta_{1}, \theta_{2}, \theta_{3},-\theta_{3}\right)}{F_{d}}
$$

We remind the reader that we use translation invariance to relate all four-point correlators to those of the form $F\left(\theta_{1}, \theta_{2}, \theta_{3},-\theta_{3}\right)$, followed by the use of anti-periodicity, swap symmetry, and $C(P) T$ invariance (see appendix B) to relate all such correlators to those with $0<\theta_{12}^{ \pm}<2 \pi$ and $0<\theta_{3}<\pi$ (a.k.a. $\theta_{34}^{+}=0$ and $0<\theta_{34}^{-}<2 \pi$ ). In addition, we see that due to the point reflection symmetry $\left(\theta_{12}^{-}, \theta_{12}^{+}\right) \rightarrow\left(2 \pi-\theta_{12}^{-}, 2 \pi-\theta_{12}^{+}\right)$, it is sufficient to determine the correlators in only one half of the above domain. Accordingly, out of the four possible uncrossed configurations in our domain $\theta_{1}>\theta_{2}>\theta_{3}, \theta_{3}>\theta_{1}>\theta_{2}$, $\theta_{1}>\theta_{2}>-\theta_{3}$, and $2 \pi-\theta_{3}>\theta_{1}>\theta_{2}$, we need only solve for those satisfying $\theta_{1}>\theta_{2}>\theta_{3}$ and $\theta_{3}>\theta_{1}>\theta_{2}$. In conclusion, the four-point correlators of the form $F\left(\theta_{1}, \theta_{2}, \theta_{3},-\theta_{3}\right)$ with $0<\theta_{12}^{ \pm}<2 \pi$ and $0<\theta_{3}<\pi$, fall into either crossed or uncrossed configurations, and completely determine the four-point correlators for all possible times.

\subsection{Time-ordered correlators and finite temperature OPEs}

If we define

$$
f(\theta) \equiv\left(1-\left(\frac{v}{2} \theta+\cot \frac{\pi v}{2}\right) \tan \left(\frac{v}{2}(\pi-\theta)\right)\right)
$$

then the ratio of the time-ordered connected and disconnected four-point correlators can be expressed as

$$
\frac{\mathcal{F}}{F_{d}}=\frac{2 \tan \frac{\pi v}{2}}{\frac{\pi v}{2}+\cot \frac{\pi v}{2}} f\left(\theta_{12}^{-}\right) \times \begin{cases}f\left(\theta_{34}^{-}\right) & \theta_{1}>\theta_{2}>\theta_{3} \\ f\left(2 \pi-\theta_{34}^{-}\right) & \theta_{3}>\theta_{1}>\theta_{2}\end{cases}
$$

where $v=v(\beta \mathcal{J})$ is determined from (2.12).

Let us now restrict attention to the configuration $\theta_{1}>\theta_{2}>\theta_{3}$. Then, by defining the bi-local flavor-averaged operators $\hat{G}_{i j} \equiv \hat{G}\left(\theta_{i}, \theta_{j}\right) \equiv \frac{1}{N} \sum_{k=1}^{N} \hat{\psi}_{k}\left(\tau_{i}\right) \hat{\psi}_{k}\left(\tau_{j}\right)$, we may re-express the uncrossed four-point function (3.3) as arising from a sum of products of three-point correlators

$$
\begin{aligned}
& F=G\left(\theta_{12}^{-}\right) G\left(\theta_{34}^{-}\right)+G\left(\theta_{12}^{-}\right) G\left(\theta_{34}^{-}\right) \frac{2 \tan \frac{\pi v}{2}}{\frac{\pi v}{2}+\cot \frac{\pi v}{2}} \frac{f\left(\theta_{12}^{-}\right) f\left(\theta_{34}^{-}\right)}{N} \\
& F=\left\langle\hat{G}_{12} \hat{G}_{34}\right\rangle=\left\langle\hat{G}_{12}\right\rangle\left\langle\hat{G}_{34}\right\rangle+\frac{\left\langle\delta \hat{H} \hat{G}_{12}\right\rangle\left\langle\delta \hat{H} \hat{G}_{34}\right\rangle}{\left\langle(\delta \hat{H})^{2}\right\rangle}
\end{aligned}
$$


where $\delta \hat{H} \equiv \hat{H}-\langle\hat{H}\rangle$ and the expressions for $\left\langle\delta \hat{H} \hat{G}_{12}\right\rangle$ may be obtained by writing $G_{12}$ in terms of $\tau$ variables and then taking derivatives with respect to $\beta$. This is entirely equivalent to performing an OPE-type expansion, which usually corresponds vacuum-expanding a bilocal product operator $\hat{\mathcal{O}}$ in a "complete basis" of local operators [10]

$$
\hat{\mathcal{O}}=\sum_{\hat{A}}\left\langle\hat{A}^{\dagger} \mathcal{O}\right\rangle \hat{A}
$$

which will relate higher correlators to products of lower correlators of some "complete basis" of operators. In this case, we take the identity operator and the Gram-Schmidt processed Hamiltonian $\delta \hat{H} \equiv \hat{H}-\langle\hat{H}\rangle$ as two unnormalized operators of our basis. Surprisingly, (3.4) then implies that these two operators are sufficient to find the asymptotic time-ordered four-point correlator at large $q$ !

One may then wonder what is occurring when $\theta_{3}>\theta_{1}>\theta_{2}(3.3)$, as the fermions are still uncrossed, but the functional form of the resultant time-ordered four-point function changes from being a simple function of $\theta_{34}^{-}$to one of $2 \pi-\theta_{34}^{-}$. That is, the instance of $\theta_{34}^{-}$in $\left\langle\delta \hat{H} \hat{G}_{34}\right\rangle$ in the OPE-like relations (3.4) must replaced by $2 \pi-\theta_{34}^{-}$(even though the correlator $\left\langle\hat{G}_{34}\right\rangle$ remains invariant under $\theta_{34}^{-} \leftrightarrow 2 \pi-\theta_{34}^{-}$). To figure out what's going on, let's write out this correlator more explicitly

$$
\begin{aligned}
F\left(\theta_{1}, \theta_{2}, \theta_{3},-\theta_{3}\right) & =\frac{1}{N} \sum_{j} \operatorname{Tr}\left(\hat{\rho} \hat{\psi}_{j}\left(\theta_{3}\right) \hat{G}_{12} \hat{\psi}_{j}\left(\theta_{4}\right)\right), & & \theta_{3}>\theta_{1}>\theta_{2} \\
& =\frac{1}{N} \sum_{j} \operatorname{Tr}\left(\hat{\rho} \hat{G}_{12} \hat{\psi}_{j}\left(\theta_{4}\right) \hat{\psi}_{j}\left(\theta_{3}-2 \pi\right)\right), & & \theta_{3}>\theta_{1}>\theta_{2} \\
& =\left\langle\hat{G}\left(\theta_{1}, \theta_{2}\right) \hat{G}\left(\theta_{4}, \theta_{3}-2 \pi\right)\right\rangle, & & \theta_{3}>\theta_{1}>\theta_{2}
\end{aligned}
$$

where the angular separation between the two times in the second operator is $\theta_{4}-$ $\left(\theta_{3}-2 \pi\right)=2 \pi-\theta_{4}+\theta_{3}=2 \pi-\theta_{34}^{-}$. At this point, we can run through the process of inserting complete sets of operators and we naturally arrive at expressions containing the angular separation $2 \pi-\theta_{34}^{-}$rather than $\theta_{34}^{-}$for when $\theta_{3}>\theta_{1}>\theta_{2}(3.3)$.

We should also note that this phenomenon where the time-ordered behavior arises from "thermal fluctuations" does not hold solely in the large $q$ limit. In fact, it has been shown that for any $q>2$, these relations will apply for the near-conformal limit of large $\beta \mathcal{J}$ [5]. Furthermore, these relations apply to any model where the fluctuations are governed by a Schwarzian action - a.k.a. a near-conformal limit - and not just the $S Y K$ model [7].

\subsection{Out-of-time-order correlators and chaos}

It will be useful to define the squeezed coordinates $\pi-\phi_{i j}^{ \pm} \equiv v\left(\pi-\theta_{i j}^{ \pm}\right)$where if $\theta_{i j}^{ \pm} \in(0,2 \pi)$, then $\phi$ will range over the squeezed domain $\phi_{i j}^{ \pm} \in \pi(1-v, 1+v)$ since $0 \leq v<1$. Using this we may define the quantity

$$
g(\phi) \equiv 1+\frac{\pi-\phi}{2 \tan \frac{\phi}{2}}
$$


which allows us to write the large $N$ behavior of the connected out-of-time-order correlator $\theta_{1}>\theta_{3}>\theta_{2}$ on one line

$$
\frac{\mathcal{F}}{F_{d}}=\frac{2 \tan \frac{\pi v}{2}}{\frac{\pi v}{2}+\cot \frac{\pi v}{2}} g\left(\phi_{12}^{-}\right) g\left(\phi_{34}^{-}\right)-\frac{2 \sin \frac{\phi_{12}^{+}}{2}}{\cos \frac{\pi v}{2} \sin \frac{\phi_{12}^{-}}{2} \sin \frac{\phi_{34}^{-}}{2}}-\frac{\tan \frac{\pi v}{2}\left(\pi-\phi_{12}^{+}\right)}{\tan \frac{\phi_{12}^{-}}{2} \tan \frac{\phi_{34}^{-}}{2}}
$$

where $v=v(\beta \mathcal{J})$ is determined from (2.12). In the limit of large $\beta \mathcal{J}$ where $v$ approaches 1 , this agrees with the large $q$ limit of previous large $\beta \mathcal{J}$ results $[5,7,8]$. Unlike previous results, this expression applies outside of the large $\beta \mathcal{J}$ regime, so long as one is at large $q$. Indeed, one may explicitly check small $\beta \mathcal{J}$ validity by expanding the first couple terms in the four-point function (A.4). In other words, this is the first expression for a large $N$ $S Y K$ four-point function that is correct both at strong and weak coupling. Particularly, we see that the entirety of the $\beta \mathcal{J}$ dependence has been absorbed into the parameter $v$. Furthermore, after going to the rescaled coordinates the four-point function only has three different types of $v$-dependence, all of which diverge linearly as $v \rightarrow 1$.

One way of defining scrambling is when the Lorentzian evolution of two operators in an out-of-time-order four-point correlator causes the overall function to deviate significantly from the disconnected correlator. In this case, since we have $F \equiv F_{d}+\mathcal{F} / N$, scrambling can be thought of as occurring when Lorentzian evolution causes $\mathcal{F} / F_{d} \sim N$. Now, as for the Euclidean positioning of the operators in a scrambling setup, there are many different configurations one can consider which preserve the crossed nature of the ordering. Regardless, one then Lorentzian evolves the system so that $\left(\tau_{1}, \tau_{2}\right) \rightarrow\left(\tau_{1}+i t, \tau_{2}+i t\right)$ which makes it so $\theta_{12}^{-}, \theta_{34}^{-}$, and $\theta_{34}^{+}$are held fixed while $\theta_{12}^{+} \rightarrow \theta_{12}^{+} \pm 4 \pi i t / \beta$. In other words, we can consider Lorentzian evolution by giving $\phi_{12}^{+} / 2$ an imaginary component $\phi_{12}^{+} / 2 \rightarrow \phi_{12}^{+} / 2+i \frac{2 \pi v}{\beta} t$. Plugging this into (3.10), we see that there are both linearly and exponentially growing terms in the out-of-time-order correlator. Keeping only the exponentially growing term, we find that

$$
\frac{\mathcal{F}}{F_{d}} \sim-\frac{e^{i \operatorname{sgn}(t)\left(\pi-\phi_{12}^{+}\right) / 2} e^{2 \pi t v / \beta}}{\cos \frac{\pi v}{2} \sin \frac{\phi_{12}^{-}}{2} \sin \frac{\phi_{34}^{-}}{2}}+\mathcal{O}(2 \pi v t / \beta)
$$

Thus, large $q$ scrambling occurs when

$$
\begin{aligned}
t_{\mathrm{scr}} & \sim \frac{\beta}{2 \pi v}\left(\log \left(N \cos \frac{\pi v}{2}\right)+\log \left(\sin \frac{\phi_{12}^{-}}{2} \sin \frac{\phi_{34}^{-}}{2}\right)\right) \\
& \sim \frac{\beta}{2 \pi v}\left(\log \left(N \cos \frac{\pi v}{2}\right)+\log \left(\cos \left(\frac{v}{2}\left(\pi-\theta_{12}^{-}\right)\right) \cos \left(\frac{v}{2}\left(\pi-\theta_{34}^{-}\right)\right)\right)\right)
\end{aligned}
$$

where we note that at large $\beta \mathcal{J}, \cos \frac{\pi v}{2} \rightarrow \frac{\pi}{\beta \mathcal{J}}\left(1-\frac{2}{\beta \mathcal{J}}+\ldots\right)$, which means that the scrambling time can be nontrivially modified depending of whether the Euclidean angle differences $\left(\theta_{12}^{-}, \theta_{34}^{-}\right)$are comparable to $1 / \beta \mathcal{J}$.

Now since $0 \leq v<1$, the Lyapunov exponent of the large $q S Y K$ model is always below the chaos bound $[6]$

$$
\lambda_{L}=\frac{2 \pi}{\beta} v<\lambda_{L}^{\text {bound }}=\frac{2 \pi}{\beta}
$$

It is interesting to consider the fact that the rate of growth of the connected out-of-timeorder four-point correlator is also governed by the thermalization timescale $t_{\text {thermal }} \sim \frac{\beta}{v}$. 
Alternatively stated, we have derived that the thermalization rate and the Lyapunov exponent are the same in the large $q S Y K$ model for all energies, even when the system is described by perturbative physics.

\section{Discussion}

It is interesting how the functional form of the large $q$ SYK out-of-time-order correlator (3.10) is universal: everything can be absorbed in a new parameter $v$, which is monotonic in the coupling and temperature (2.12). Going to low temperature/strong coupling, one may check that it matches the previously-derived low-temperature near-conformal expression $[5,7,8]$ and has therefore re-summed certain corrections sub-leading in $\beta \mathcal{J}$.

Originally, such terms arise from the kinetic term's breaking of the low-temperature conformal symmetry of the SYK action, which manifests in the saddle-point equation as a derivative (or $\left[G_{0}\right]$ in Matsubara space) (2.2). Now, this breaking has not disappeared in the large $q$ limit and manifests as a constraint on the resultant large $q$ Liouville theory that would otherwise be conformal $[5,9]$ : the kinetic term in the original action restricts the Liouville field variable and its fluctuations to only those that are zero at zero time difference. Thus, it is still true that at large $q$ one may think of the low energy physics as being described by a spontaneously and explicitly broken conformal symmetry. In other words, taking the large $q$ limit preserves many interesting properties of the $S Y K$ model, yet makes the theory as a whole much more tractable.

We remind the reader of our result that the large $q$ thermalization rate and scrambling rate (Lyapunov exponent) are the same at all couplings and energies. This is quite significant, since the two correspond to different physics. From perspective of operator dynamics, the thermalization time is when an operator has appreciably changed and become anything else; in contrast, the scrambling time is when an operator has become everything else. More specifically, the process of scrambling as defined by OTO decay refers to the increasing non-commutativity between some evolving operator with some simple reference operator(s) [15-17]. Now, characterizing how "much" an operator does not commute with some reference operator(s) orders the space of operators into "smaller" and "larger" operators; it is in this sense that that the Lyapunov exponent is the rate of "operator growth" $[10,12]$. It is thus clear that the rates of operator change (thermalization) and growth (scrambling) need not be the same, hence why it is so interesting that these $q$-local interactions regardless of energy or coupling cause these two kinds of operator dynamics to occur at the same rate.

The infinite temperature epidemic picture obtained by repeatedly applying commutations of the Hamiltonian to a simple operator naturally produces such a prediction [12]. The fact that the agreement between these two rates occurs at all energies suggests that at any energy, some form of thermally renormalized epidemic model applies [10]. Furthermore, there is a growing body of evidence that holographic OTOs are expectation values of bulk (null) momenta for boundary-perturbed states $\hat{\mathcal{O}}(t)|\mathrm{TFD}\rangle[7,18,19]$. In other words, the operator dynamics of holographic theories seems to be closely related to classical mechanics on black hole geometries [10,11, 20-23]. Having a specific understanding of 
how this occurs would greatly aid in explaining the relation between semi-classical gravity and large $N$ quantum mechanics. We are only now beginning to learn about the smooth and simple structures that underly the operator dynamics of $q$-local theories [24].

\section{Acknowledgments}

We are grateful to Adam Levine, Henry Lin, Andy Lucas, Juan Maldacena, Phil Saad, Steve Shenker, Douglas Stanford, Lenny Susskind, and Ying Zhao for useful discussions. AS is supported by the Simons Foundation.

Research at Perimeter Institute is supported in part by the Government of Canada through the Department of Innovation, Science and Economic Development Canada and by the Province of Ontario through the Ministry of Economic Development, Job Creation and Trade.

\section{A Twisting correlators}

First, we temporarily purify the system. This will (often implicitly) involve the introduction of a maximally entangled state $|0\rangle$ that satisfies

$$
\left(A_{L}-e^{i \varphi_{A}} A_{R}\right)|0\rangle=0
$$

for all "basic" operators $(A, B$, etc. $){ }^{1}$ That is, we define $|0\rangle$ as the vacuum under the "annihilation operators" such as $c_{A} \equiv A_{L}+e^{i \varphi_{A}} A_{R}$; thus, we see that $|0\rangle$ is analogous to a state of simple bell pairs. Suppose that we want the four-point function $\langle A B C D\rangle_{\beta}$ with $\pi>\theta_{A}>\theta_{B}>\theta_{C}>0$ and $\theta_{D}=-\theta_{C}$, then we have that $\langle A B C D\rangle_{\beta} \propto\left\langle A_{L} B_{L} C_{L} D_{R}\right\rangle_{\mathrm{TFD}}$ where the proportionality factor is a simple phase. For ensembles of four-point functions, we have $\sum_{C D} w_{C} w_{D}\langle A B C D\rangle_{\beta} \propto \sum_{C D} w_{C} w_{D}\left\langle A_{L} B_{L} C_{L} D_{R}\right\rangle_{\mathrm{TFD}}$.

At this point, we have yet to do anything particularly non-trivial; the crux is the next step. There exists choices of $(C, D)$ and weightings $\left(w_{C}, w_{D}\right)$ such that the doubled operator

$$
V \propto \sum_{C D} w_{C} w_{D} C_{L} D_{R}
$$

generates twists of doubled coherent states: $\left[V, a_{L}\right]=V_{L L} a_{L}+V_{L R} a_{R}$ and $\left[V, a_{R}\right]=V_{R L} a_{L}+$ $V_{R R} a_{R}$ where $a_{L / R}$ are annihilation operators. Consequently, $M \equiv e^{-\mu V}$ maps one doubled coherent state into another: $M\left|\alpha_{L}, \alpha_{R}\right\rangle=\left|M_{L L} \alpha_{L}+M_{L R} \alpha_{R}, M_{R L} \alpha_{L}+M_{R R} \alpha_{R}\right\rangle$. Since path integrals ${ }^{2}$ are constructed from coherent states, the effect of $M$ is solely to provide a twist condition on the fields. If we then calculate the correlator $\mathcal{G}_{A B} \equiv\left\langle A_{L} B_{L} M\right\rangle_{\mathrm{TFD}}$ with the twist conditions applying to all configurations, then we may then obtain the fourpoint function via $\lim _{\mu \rightarrow 0} \partial_{\mu} \mathcal{G}_{A B}=\left\langle A_{L} B_{L} V\right\rangle_{\mathrm{TFD}} \propto \sum_{C D} w_{C} w_{D}\langle A B C D\rangle_{\beta}$. At this point,

\footnotetext{
${ }^{1}$ Note that if $\left[A_{L}, B_{L}\right]_{\xi}=C_{L}$, then (A.1) implies that $\left[A_{R}, B_{R}\right]_{\xi}=-e^{i\left(\varphi_{C}-\varphi_{A}-\varphi_{B}\right)} C_{R}$.

${ }^{2}$ Note that typically path integrals of bosonic degrees of freedom such as $\phi(x)$ are often not often expressed in terms of $\alpha(x) \equiv \phi(x)+i \pi(x)$, since we usually integrate out $\pi(x)$. However, it is not difficult to first translate the $\alpha(x)$ twist conditions into twist conditions on $\phi(x)$ and $\pi(x)$ before attempting to integrate out $\pi(x)$.
} 
we need not continue to work in the purified system, as we may de-purify by sewing the left-right configuration integrals together by defining the continuous field:

$$
A(\theta)= \begin{cases}A_{L}(\theta) & 0 \leq \theta \leq \pi \\ -e^{i \varphi_{A}} A_{R}(2 \pi-\theta) & \pi \leq \theta<2 \pi\end{cases}
$$

This allows us to cast the doubled twist conditions in terms of the original system. We thereby conclude that to determine (weighted averages of) four-point functions that can be cast as $\left\langle A_{L} B_{L} V\right\rangle_{\mathrm{TFD}}$ for $V$ which generates $L R$ coherent state twists, one may instead calculate the $A B$ two-point function, with the restriction on the path integral that all configurations obey de-purified twist conditions.

Suppose we are interested in the finite temperature four-point function involving two pairs of Hermitian fermionic operators

$$
F\left(\theta_{1}, \theta_{2}, \theta_{3}, \theta_{4}\right)=\frac{1}{N^{2}} \sum_{i, j=1}^{N}\left\langle\mathcal{T}\left[\psi_{i}\left(\theta_{1}\right) \psi_{i}\left(\theta_{2}\right) \psi_{j}\left(\theta_{3}\right) \psi_{j}\left(\theta_{4}\right)\right]\right\rangle_{\beta}
$$

where $\mathcal{T}$ is the Euclidean time-ordering symbol and $\theta \equiv 2 \pi \tau / \beta$. Defining the coordinates $\theta_{i j}^{ \pm} \equiv \theta_{i} \pm \theta_{j}$, we use time-translation invariance to set $\theta_{34}^{+}=0$. Thus, without loss of generality we take $\theta_{3}=-\theta_{4}=\theta_{34}^{-} / 2$. As discussed in appendix (B), this choice makes it clear that for $C(P) T$-invariant systems it is sufficient to solve for $F_{i i j j}$ in one half of the $\left(\theta_{1}, \theta_{2}\right)$ domain defined by $0<\theta_{12}^{ \pm}<2 \pi$ and $0<\theta_{34}^{-}<2 \pi$. In other words, we need only study one-eighth of the typical fundamental domain. Essentially, each of the operator pairings as well as the $C(P) T$ invariance cuts the domain in half.

With the domain restricted, we need to figure out how to calculate $F$. In our case, it will be extremely useful to temporarily purify the calculation via the maximally entangled state $|0\rangle,{ }^{3}$ which satisfies $\left(\psi_{i}^{L}+i \psi_{i}^{R}\right)|0\rangle=0$. We find

$$
F\left(\theta_{1}, \theta_{2}, \theta_{3},-\theta_{3}\right)=-\frac{i}{N^{2}} \sum_{i, j=1}^{N}\left\langle 0\left|\mathcal{T}\left[\rho \psi_{i}^{L}\left(\theta_{1}\right) \psi_{i}^{L}\left(\theta_{2}\right) \psi_{j}^{L}\left(\theta_{3}\right) \psi_{j}^{R}\left(-\theta_{3}\right)\right]\right| 0\right\rangle
$$

where $\mathcal{T}$ is the contour-ordering symbol and $\rho$ is the purified Gibbs state such that $\rho^{1 / 2}|0\rangle=$ $|\mathrm{TFD}\rangle: \rho=e^{-\beta\left(H_{L}+H_{R}\right) / 2} / Z(\beta)$.

We then proceed to study a more complicated object

$$
\mathcal{Z}^{\prime} \equiv \frac{1}{N} \sum_{i=1}^{N}\left\langle 0\left|\mathcal{T}\left[\rho \psi_{i}^{L}\left(\theta_{1}\right) \psi_{i}^{L}\left(\theta_{2}\right) M\left(\theta_{3}\right)\right]\right| 0\right\rangle \quad M \equiv \exp \left(-\frac{i \mu}{2} \sum_{j=1}^{N} \psi_{j}^{L} \psi_{j}^{R}\right)
$$

where $M$ is of the form $M=e^{-\mu V}$ with $V$ hermitian. We see that this object generates the four-point function: $\frac{2}{N} \lim _{\mu \rightarrow 0} \partial_{\mu} \mathcal{Z}^{\prime}=F$. Then, we note that it naturally factorizes into

\footnotetext{
${ }^{3}$ There is no unique maximally entangled state, since entanglement is unchanged by local unitaries. Thus, let us choose the simplest state $|\tilde{0}\rangle$ such that the left and right algebras are the same. To guarantee this, we start by noting that if $\left(\psi_{i}^{L}+c_{i} \psi_{i}^{R}\right)|\tilde{0}\rangle=0$, then one has that $2 \delta_{i j}|0\rangle=\left\{\psi_{i}^{L}, \psi_{j}^{L}\right\}|0\rangle=-c_{i} c_{j}\left\{\psi_{i}^{R}, \psi_{j}^{R}\right\}|0\rangle$, which gives that $c_{i} c_{j}\left\{\psi_{i}^{R}, \psi_{j}^{R}\right\}=-2 \delta_{i j}$. One is then naturally led to the choice $c_{i}= \pm i$, where in our case we chose $c_{i}=+i$. Of course, the ambiguity in this choice has no effect on physical predictions.
} 
the product

$$
\begin{aligned}
\mathcal{Z}^{\prime} & =\mathcal{Z} \mathcal{G}_{L L}\left(\theta_{1}, \theta_{2}\right) \\
\mathcal{Z} & \equiv\left\langle 0\left|\mathcal{T}\left[\rho M\left(\theta_{3}\right)\right]\right| 0\right\rangle \quad \mathcal{G}_{L L}\left(\theta_{1}, \theta_{2}\right) \equiv \frac{\mathcal{Z}^{-1}}{N} \sum_{i=1}^{N}\left\langle 0\left|\mathcal{T}\left[\rho \psi_{i}^{L}\left(\theta_{1}\right) \psi_{i}^{L}\left(\theta_{2}\right) M\left(\theta_{3}\right)\right]\right| 0\right\rangle
\end{aligned}
$$

Furthermore, by taking derivatives and setting $\mu \rightarrow 0$, we see that this factorization implies that

$$
\begin{aligned}
\frac{2}{N} \lim _{\mu \rightarrow 0} \partial_{\mu} \mathcal{Z}^{\prime} & =\frac{2}{N} \lim _{\mu \rightarrow 0}\left(\partial_{\mu} \mathcal{Z}\right) \mathcal{G}+\frac{2}{N} \lim _{\mu \rightarrow 0} \mathcal{Z}\left(\partial_{\mu} \mathcal{G}\right) \\
\Rightarrow F\left(\theta_{1}, \theta_{2}, \theta_{3},-\theta_{3}\right) & =-i G_{L L}\left(\theta_{12}^{-}\right) G_{L R}\left(\theta_{34}^{-}\right)+\frac{2}{N} \lim _{\mu \rightarrow 0} \partial_{\mu} \mathcal{G}_{L L}\left(\theta_{1}, \theta_{2}\right)
\end{aligned}
$$

Thus, if we are able to determine the regular two-point functions $G_{L L}$ and $G_{L R}$, as well as the twisted two-point function $\mathcal{G}_{L L}$ to leading order in $\mu$, then we have obtained the full four-point function.

As discussed in [10], the role of $M=\exp \left(-\frac{i \mu}{2} \sum_{j=1}^{N} \psi_{j}^{L} \psi_{j}^{R}\right)$ in (A.7) is solely to twist the boundary condition of the fermions from being continuous as one approaches $\theta_{0}$ from either direction to

$$
\lim _{\theta \rightarrow \theta_{3}^{+}}\left(\begin{array}{c}
\psi^{L}(\theta) \\
i \psi^{R}(\theta)
\end{array}\right)=\left(\begin{array}{cc}
\cosh (\mu) & -\sinh (\mu) \\
-\sinh (\mu) & \cosh (\mu)
\end{array}\right) \lim _{\theta \rightarrow \theta_{3}^{-}}\left(\begin{array}{c}
\psi^{L}(\theta) \\
i \psi^{R}(\theta)
\end{array}\right)
$$

If we then de-purify the calculation by stitching the left and right fields together

$$
\psi(\theta)= \begin{cases}\psi_{L}(\theta) & 0 \leq \theta \leq \pi \\ i \psi_{R}(2 \pi-\theta) & \pi \leq \theta<2 \pi\end{cases}
$$

making sure carry along our boundary conditions (A.9) which become

$$
\lim _{\theta \rightarrow \theta_{3}^{+}}\left(\begin{array}{c}
\psi(\theta) \\
\psi(2 \pi-\theta)
\end{array}\right)=\left(\begin{array}{cc}
\cosh (\mu) & -\sinh (\mu) \\
-\sinh (\mu) & \cosh (\mu)
\end{array}\right) \lim _{\theta \rightarrow \theta_{3}^{-}}\left(\begin{array}{c}
\psi(\theta) \\
\psi(2 \pi-\theta)
\end{array}\right)
$$

then we obtain we see that our four-point function relation (A.8) becomes

$$
F\left(\theta_{1}, \theta_{2}, \theta_{3},-\theta_{3}\right)=G\left(\theta_{12}^{-}\right) G\left(\theta_{34}^{-}\right)+\frac{2}{N} \lim _{\mu \rightarrow 0} \partial_{\mu} \mathcal{G}\left(\theta_{1}, \theta_{2}\right)
$$

where $\mathcal{G}$ is now a two point function on a single Hilbert space, satisfying the bi-local twist conditions (A.11). Thus, we conclude that the factorization (A.7) that was natural in the doubled theory is actually large $N$ factorization.

Defining

$$
F=F_{d}+\frac{\mathcal{F}}{N}
$$

where $F_{d} \equiv G\left(\theta_{12}^{-}\right) G\left(\theta_{34}^{-}\right)$, then we see that the connected part of the four-point function is given by

$$
\mathcal{F}\left(\theta_{1}, \theta_{2}, \theta_{3},-\theta_{3}\right)=2 \lim _{\mu \rightarrow 0} \partial_{\mu} \mathcal{G}\left(\theta_{1}, \theta_{2}\right)
$$


Oftentimes, we are more concerned with the ratio of the connected and disconnected fourpoint functions, which is given by

$$
\frac{\mathcal{F}\left(\theta_{1}, \theta_{2}, \theta_{3},-\theta_{3}\right)}{F_{d}}=\frac{2}{G\left(\theta_{34}^{-}\right)} \lim _{\mu \rightarrow 0} \partial_{\mu} \ln \mathcal{G}\left(\theta_{1}, \theta_{2}\right)
$$

For this reason, we will switch variables to $\nu \equiv \mu G\left(\theta_{34}^{-}\right)$before taking our limits:

$$
\frac{\mathcal{F}\left(\theta_{1}, \theta_{2}, \theta_{3},-\theta_{3}\right)}{G\left(\theta_{12}^{-}\right) G\left(\theta_{34}^{-}\right)}=2 \lim _{\nu \rightarrow 0} \partial_{\nu} \ln \mathcal{G}\left(\theta_{1}, \theta_{2}\right)
$$

\section{B Fundamental domain of four-point functions}

A four-point function of two pairs of operators has much more symmetry than a generic four-point function, which will we use to vastly restrict the fundamental domain.

First, we note that $F_{i i j j}$ is odd when the first pair of times $\left(\tau_{1}, \tau_{2}\right)$ are reflected across the lines $\tau_{1}-\tau_{2}=n \beta \quad \forall n \in \mathbb{Z}$

$$
F_{i i j j}\left(\tau_{2}+n \beta, \tau_{1}-n \beta, \tau_{0}\right)=-F_{i i j j}\left(\tau_{1}, \tau_{2}, \tau_{0}\right)
$$

Thus, knowledge of the four-point function for $0<\tau_{1}-\tau_{2}<\beta$ is sufficient to determine it everywhere. A similar condition on $\tau_{3}$ and $\tau_{4}$ implies that we may use as a fundamental domain $0<\tau_{3}-\tau_{4}<\beta$, which for our parametrization implies that we need only understand $F_{i i j j}\left(\tau_{1}, \tau_{2}, \tau_{0}\right)$ for $0<\tau_{0}<\beta / 2$ to determine the four-point function everywhere.

Second, $C(P) T$ invariance implies that $F_{i i j j}\left(\tau_{1}, \tau_{2}, \tau_{3}, \tau_{4}\right)$ satisfies

$$
\begin{aligned}
F_{i i j j}\left(\tau_{1}, \tau_{2}, \tau_{3}, \tau_{4}\right) & =F_{j j i i}\left(\beta-\tau_{4}, \beta-\tau_{3}, \beta-\tau_{2}, \beta-\tau_{1}\right) \\
& =F_{i i j j}\left(\beta-\tau_{1}, \beta-\tau_{2}, \beta-\tau_{3}, \beta-\tau_{4}\right) \\
& =F_{i i j j}\left(\beta-\tau_{2}, \beta-\tau_{1}, \beta-\tau_{4}, \beta-\tau_{3}\right)
\end{aligned}
$$

Now we see that with $\hat{G}_{12}$ our choice $\tau_{3}=\beta-\tau_{0}, \tau_{4}=\tau_{0}$, we have the relationship

$$
F_{i i j j}\left(\tau_{1}, \tau_{2}, \tau_{0}\right)=F_{i i j j}\left(\beta-\tau_{2}, \beta-\tau_{1}, \tau_{0}\right)=F\left(\beta-\tau_{1},-\tau_{2}, \tau_{0}\right)
$$

Combined with anti-periodicity of $F_{i i j j}$ under $\beta$ translations of $\tau_{1}$ and $\tau_{2}$, we conclude that $F_{i i j j}$ is even when $\left(\tau_{1}, \tau_{2}\right)$ are reflected across the lines $\tau_{1}+\tau_{2}=m \beta \quad \forall m \in \mathbb{Z}$. Consequently, we may restrict our domain of $F\left(\tau_{1}, \tau_{2}, \tau_{0}\right)$ to $0<\tau_{1}+\tau_{2}<\beta$. In fact, we may actually restrict this further, since we note that composing the reflections and translations produces non-trivial point reflections. Specifically, the second equality in (B.5) tells us that $F$ is even under reflections through the point $\left(\tau_{1}=\beta / 2, \tau_{2}=0\right)$. We thereby conclude that it is sufficient to solve for $F_{i i j j}$ in one half of the $\left(\tau_{1}, \tau_{2}\right)$ domain defined by $0<\tau_{1} \pm \tau_{2}<\beta$ as well as for $\tau_{3}=\beta-\tau_{0}, \tau_{4}=\tau_{0}$, with $\tau_{0}<\beta / 2$ to determine $F_{i i j j}$ everywhere.

Note that the fundamental domain for two pairs of operators is only one-eighth of the fundamental domain for a generic four-point functions. This factor came from a halving of the fundamental domain due to each of the swap symmetries, and a final halving due to the $C(P) T$ symmetry. 
Open Access. This article is distributed under the terms of the Creative Commons Attribution License (CC-BY 4.0), which permits any use, distribution and reproduction in any medium, provided the original author(s) and source are credited.

\section{References}

[1] S. Sachdev and J. Ye, Gapless spin fluid ground state in a random, quantum Heisenberg magnet, Phys. Rev. Lett. 70 (1993) 3339 [cond-mat/9212030] [INSPIRE].

[2] A. Kitaev, Hidden correlations in the hawking radiation and thermal noise, talk given at the Fundamental Physics Prize Symposium, November 10 (2014).

[3] I.R. Klebanov and G. Tarnopolsky, Uncolored random tensors, melon diagrams, and the sachdev-ye-kitaev models, Phys. Rev. D 95 (2017) 046004.

[4] A. Kitaev, A simple model of quantum holography, talks given at KITP, April 7 and May 27 (2015).

[5] J. Maldacena and D. Stanford, Remarks on the Sachdev-Ye-Kitaev model, Phys. Rev. D 94 (2016) 106002 [arXiv:1604.07818] [INSPIRE].

[6] J. Maldacena, S.H. Shenker and D. Stanford, A bound on chaos, JHEP 08 (2016) 106 [arXiv: 1503.01409] [INSPIRE].

[7] J. Maldacena, D. Stanford and Z. Yang, Conformal symmetry and its breaking in two-dimensional nearly Anti-de Sitter space, Prog. Theor. Exp. Phys. (2016) 12C104.

[8] A. Kitaev and S.J. Suh, The soft mode in the Sachdev-Ye-Kitaev model and its gravity dual, JHEP 05 (2018) 183.

[9] J. Maldacena and X.-L. Qi, Eternal traversable wormhole, arXiv:1804.00491 [INSPIRE].

[10] X.-L. Qi and A. Streicher, Quantum epidemiology: operator growth, thermal effects and SYK, JHEP 08 (2019) 012 [arXiv: 1810.11958] [INSPIRE].

[11] A.R. Brown et al., Falling toward charged black holes, Phys. Rev. D 98 (2018) 126016 [arXiv: 1804.04156] [INSPIRE].

[12] D.A. Roberts, D. Stanford and A. Streicher, Operator growth in the SYK model, JHEP 06 (2018) 122 [arXiv: 1802.02633] [INSPIRE].

[13] D.A. Roberts and D. Stanford, Two-dimensional conformal field theory and the butterfly effect, Phys. Rev. Lett. 115 (2015) 131603 [arXiv:1412.5123] [INSPIRE].

[14] D.J. Gross and V. Rosenhaus, A Generalization of Sachdev-Ye-Kitaev, JHEP 02 (2017) 093 [arXiv: 1610.01569] [INSPIRE].

[15] D. Stanford, Many-body chaos at weak coupling, JHEP 10 (2016) 009 [arXiv:1512.07687] [INSPIRE].

[16] S.H. Shenker and D. Stanford, Black holes and the butterfly effect, JHEP 03 (2014) 067 [arXiv: 1306.0622] [INSPIRE].

[17] N. Lashkari et al., Towards the fast scrambling conjecture, JHEP 04 (2013) 022 [arXiv:1111.6580] [INSPIRE].

[18] J. Maldacena, D. Stanford and Z. Yang, Diving into traversable wormholes, Fortsch. Phys. 65 (2017) 1700034 [arXiv:1704.05333] [INSPIRE]. 
[19] S.H. Shenker and D. Stanford, Stringy effects in scrambling, JHEP 05 (2015) 132 [arXiv: 1412.6087] [INSPIRE].

[20] A. Mousatov, Operator size for holographic field theories, arXiv:1911.05089 [INSPIRE].

[21] H.W. Lin, J. Maldacena and Y. Zhao, Symmetries near the Horizon, JHEP 08 (2019) 049 [arXiv: 1904.12820] [INSPIRE].

[22] L. Susskind, Complexity and Newton's Laws, arXiv:1904.12819 [inSPIRE].

[23] H.W. Lin and L. Susskind, Complexity geometry and Schwarzian dynamics, JHEP 01 (2020) 087 [arXiv: 1911.02603] [INSPIRE].

[24] A. Lucas, Non-perturbative dynamics of the operator size distribution in the Sachdev-Ye-Kitaev model, arXiv:1910.09539 [INSPIRE]. 